\title{
The Prevalence and Estimates of the Cumulative INCIDENCE OF HOSPITAL-ACQUIRED INFECTIONS Among Patients Admitted to Auckland District Health Board Hospitals in New Zealand
}

\author{
Nicholas Graves, PhD; Tanya M. Nicholls, NZRCpN, DPH, CIC; Christopher G. S. Wong, MBChB; Arthur J. Morris, MD, DipABMM
}

\begin{abstract}
OBJECTIVES: To report the pooled results of seven prevalence surveys of hospital-acquired infections conducted between November 1996 and November 1999, and to use the data to predict the cumulative incidence of hospital-acquired infections in the same patient group.

DESIGN: The summary and modeling of data gathered from the routine surveillance of the point prevalence of hospitalacquired infections.

SETTING: Auckland District Health Board Hospitals (Auckland DHBH), the largest publicly funded hospital group in New Zealand supplying secondary and tertiary services.

PATIENTS: All inpatients.

METHOD: Point-prevalence surveys were conducted including all patients in Auckland DHBH. Standard definitions of hospital-acquired infection were used. The data from the seven surveys were pooled and used in a modeling exercise to predict
\end{abstract}

the cumulative incidence of hospital-acquired infection. An existing method for the conversion of prevalence to cumulative incidence was applied. Results are presented for all patients and stratified by clinical service and site of hospital-acquired infection.

RESULTS: The underlying patterns of hospital-acquired infection by site and service were stable during the seven time periods. The prevalence rate for all patients was $9.5 \%$, with 553 patients identified with one or more hospital-acquired infections from a population of 5,819 . The predicted cumulative incidence for all patients was $6.33 \%$ (95\% confidence interval, $6.20 \%$ to $6.46 \%$ ).

CONCLUSIONS: The prevalence and the predicted cumulative incidence are similar to rates reported in the international literature. The validity of the predicted cumulative incidence derived here is not known. If it were accurate, then the application of this method would represent a cost-effective alternative to incidence studies (Infect Control Hosp Epidemiol 2003;24:56-61).
Hospital-acquired infection imposes costs on hospitals, community care services, and patients and their families. When patients and their families are delayed in their return to productive activities, losses in productivity also accrue. ${ }^{1}$ Hospital-acquired infection also has an impact on the mortality rate. Data from the United States suggest that $10 \%$ of patients with a hospital-acquired infection die in the hospital. The hospital-acquired infection is the main cause in $10 \%$ of these deaths and a contributory factor in another $30 \%{ }^{2}$ It has been argued that the economic benefits of prevention are likely to exceed the costs. ${ }^{3.5}$ Data on the costs of hospital-acquired infection and the cost-effectiveness of prevention programs should be made available to policymakers. However, before these data can be procured, it is necessary to understand the size and the extent of the problem.

Point-prevalence surveys have been conducted twice a year, in May and November, since 1996 in Auckland District Health Board Hospitals. The results of the first survey have been published and showed that $12 \%$ of patients had a hospital-acquired infection on the day of the survey. ${ }^{6}$ Because hospital-acquired infection prolongs the hospital stay, the likelihood of such patients' being sampled in a prevalence survey increases; thus, the prevalence rate may not provide a true picture of the pattern of disease over time. A more useful measure of hospital-acquired infection is the cumulative incidence, because this illustrates the number of patients who acquire a hospital-acquired infection during a defined time period (eg, a year) and the number of cases may be expressed as a percentage of total discharges. Incidence studies are expensive to conduct, because data have to be collected on every new admission for a defined period.

Auckland District Health Board Hospitals is New Zealand's largest publicly funded, tertiary healthcare provider, with four specialist teaching hospitals treating a total of 95,999 inpatients annually. Three of the four hospitals are included in this study. Auckland Hospital has 560

Dr. Graves is from the Centre for Health Care Related Infection Surveillance and Prevention, Princess Alexandra Hospital, and the School of Public Health, Queensland University of Technology, Queensland, Australia. Ms. Nicholls is from the Auckland District Health Board, Auckland, New Zealand. Mr. Wong is from the Middlemore Hospital Auckland, Auckland, New Zealand. Dr. Morris is from the Auckland District Health Board, Infection Control Service, Auckland, New Zealand.

Address reprint requests to Dr. Nicholas Graves, School of Public Health, QUT, Victoria Park Road, Kelvin Grove, QLD, 4059, Australia.

The authors thank infectious disease physicians and registrars, clinical microbiologists and registrars, and the infection control nurse practitioners for the Auckland District Health Board Hospitals for data collection; Josephine Power for data entry; and Jenny Connor, Elizabeth Robinson, and Roger Marshall, Department of Community Health, University of Auckland, for advice on hospital epidemiology and statistical analysis. 
beds and provides emergency, acute, and elective general medical and surgical services, as well as major hematology, oncology, and liver transplant services. Green Lane Hospital has 197 beds and provides otorhinolaryngology, cardiology, respiratory medicine, and cardiothoracic surgery services, including heart and lung transplants. National Women's Hospital has 180 beds, delivers approximately 7,500 infants per year, and provides neonatal intensive care and gynecologic services. The fourth hospital was a specialist pediatrics hospital whose patients were not included in any of the prevalence surveys.

This article reports the results of seven prevalence studies conducted between November 1996 and November 1999, and derives an estimate of the cumulative incidence of hospital-acquired infection from these prevalence data. The method used ${ }^{7}$ was previously applied in two studies and found to produce valid results. ${ }^{8,9}$

\section{METHODS}

\section{Prevalence Survey}

The methods used for the prevalence surveys have been reported in detail elsewhere. ${ }^{6}$ In summary, all inpatients at the three sites of Auckland District Health Board Hospitals were included. Data collection was by twoperson teams possibly including a clinical microbiologist, an infectious diseases physician, and an infection control nurse. The clinical records of each patient were examined, and, if required, additional data were collected from the team caring for the patient. The following demographic and clinical data were recorded on a standardized data collection form: age, gender, admission date, hospital, ward, clinical service, duration of stay, presence of infection on admission, whether infection was related to a previous admission, infection present or under treatment on day of survey, site of infection, and causative organism. In addition, data were recorded on extrinsic risk factors. The definitions of the Centers for Disease Control and Prevention were used to identify the presence of hospital-acquired infection. ${ }^{10}$ The numbers of hospital-acquired infections observed on the day of the survey, which related to that admission, were divided by the number of inpatients on that day to derive a measure of the prevalence.

\section{Conversion of Prevalence Data to Estimates of Cumulative Incidence}

The formula

$$
I=P\left(\frac{L A}{L N-I N T}\right)
$$

is used to describe the relationship between prevalence and cumulative incidence that has been proposed. ${ }^{7}$ In this formula, $I$ stands for cumulative incidence, $P$ for prevalence, $L A$ for average length of stay of all patients, $L N$ for average length of stay of patients who acquire one or more nosocomial infections, and INT for average interval between admission and onset of the first nosocomial infec-
TABLE 1

Estimated MEAn Duration of Treatment for HospitalACQUIRED INFECTION, BY STTE OF HOSPITAL-ACQUIRED INFECTION

\begin{tabular}{lc}
\hline $\begin{array}{l}\text { Site of Hospital- } \\
\text { Acquired Infection }\end{array}$ & $\begin{array}{c}\text { Mean Duration } \\
\text { of Treatment (d) }\end{array}$ \\
\hline SW & 10 \\
Chest & 14 \\
UT & 5 \\
BS & 14 \\
Skin & 7 \\
MULTI & 7 \\
OTHER & 7
\end{tabular}

$\mathrm{SW}=$ surgical wound; $\mathrm{UT}$ = urinary tract; $\mathrm{BS}$ * bloodstream; MULTI = multiple sites; OTHER = other site.

Note: Lang ${ }^{17}$ is the source of all data included.

tion for those patients who acquire one or more nosocomial infections.

\section{Data Sources}

Values for $P$ were derived from the seven prevalence surveys conducted in the Auckland District Health Board Hospitals. ${ }^{6}$ Values for $L A$ were derived from data on the length of stay of every patient discharged from the relevant service in the time period. Values for $L N$ were derived from the appropriate prevalence survey. Data on length of stay were provided by the information services of the hospitals. Values for INT were not collected during these surveys. To compensate, the dates of admission and survey were compared for each patient. On the survey day, it was assumed that patients with hospital-acquired infection were halfway through a course of treatment (the estimated durations of treatment for the different sites of hospitalacquired infection are presented in Table 1) and that the onset of the infection was the day before the treatment commenced.

If the time from admission to survey was less than or equal to half the duration of treatment minus 1 day, then it was assumed that the infection was acquired on the first day of the hospital stay. On the basis of these assumptions, values for INT were derived for all patients with a hospitalacquired infection.

\section{Data Analysis}

The results of the surveys were pooled so that when the data were stratified by site of hospital-acquired infection and clinical service, the number of cases of hospitalacquired infection in each subgroup would be maximized. This required that the prevalence of hospital-acquired infection, for the different sites and the different clinical services, remain constant during the seven time periods. To demonstrate this, a general log linear regression model was developed and tested. The outcome variable was the prevalence of hospital-acquired infection. The explanatory 
TABLE 2

log linear Analysis on Prevalence Rate of Hosprtal-Acquired Infection for Main Effects and interaction Terms

\begin{tabular}{|c|c|c|c|c|c|c|}
\hline Independent Varlable & NDF & DOF & $\mathbf{F}$ & $\boldsymbol{P}>\boldsymbol{F}$ & Chl-square & P > Chl-square \\
\hline Site of hospital-acquired infection & 6 & 168 & 2.10 & $.05^{\star}$ & 12.62 & $.04^{*}$ \\
\hline Clinical service & 4 & 168 & 23.71 & $<.01^{\star}$ & 94.84 & $<.01^{\star}$ \\
\hline Time period & 6 & 168 & 0.19 & .97 & 1.17 & 0.97 \\
\hline $\begin{array}{l}\text { Time period with site of hospital- } \\
\text { acquired infection }\end{array}$ & 36 & 168 & 0.93 & .57 & 33.76 & .57 \\
\hline Time period with clinical service $^{\dagger}$ & 24 & 168 & 0.68 & .86 & 16.33 & .87 \\
\hline
\end{tabular}

$\mathrm{NDF}=$ numerator degrees of freedom; $\mathrm{DOF}=$ denominator degrees of freedom; $\mathrm{F}=\mathrm{F}$ statistic for testing the significance of the effect; $P>\mathrm{F}=P$ value based on the $\mathrm{F}$ distribution; $P>$ Chi-square $=P$ value computed from the chi-square distribution with numerator degrees of freedom.

*Significant at the $5 \%$ level.

The variable is an interaction term.

TABLE 3

Log Linear analysis on Prevalence Rate of Hospital-Acquired Infection for Main Effects Only

\begin{tabular}{lcccccc}
\hline Independent Varlable & NDF & DOF & F & $\boldsymbol{P}>$ F & Chl-square & $\boldsymbol{P}>$ Chl-square \\
\hline $\begin{array}{l}\text { Site of hospital-acquired } \\
\text { infection }\end{array}$ & 6 & 228 & 2.00 & .06 & 12.01 & \\
Clinical service & 4 & & & & & \\
Time period & 4 & 228 & 24.86 & $<.00^{*}$ & 99.47 & $0.00^{*}$ \\
& & 228 & 0.31 & .93 & 1.87 & .93
\end{tabular}

$\mathrm{NDF}=$ numerator degrees of freedom; $\mathrm{DOF}=$ denominator degrees of freedom; $\mathrm{F}=\mathrm{F}$ statistic for testing the significance of the effect; $P>\mathrm{F}=P$ value based on the $\mathrm{F}$ distribution; $P>\mathrm{Chi}-\mathrm{square}=P$ value computed from the chi-square distribution with numerator degrees of freedom.

*Significant at the $5 \%$ level.

variables were "site of hospital-acquired infection," "clinical service," "time period," and the interaction of "time period" with "site of hospital-acquired infection" and the interaction of "time period" with "clinical service." A Poisson distribution was assumed, the offset term used was the log of the population, and a dscale term was included.

The formula ${ }^{7}$ was applied to the pooled data for $P$, $L N, L A$, and $I N T$. Because values for $L N, L A$, and $I N T$ were derived from a distribution, $95 \%$ confidence intervals could be estimated. The appendix details the method used.

\section{RESULTS}

The results of the general log linear regression model are presented in Table 2.

The interactions of "site of hospital-acquired infection" and "clinical service" with "time period" were not significant ( $P=.57$ and $P=.86$, respectively), implying that the prevalence for different services and sites did not change over time. To test the main effects, the model was re-estimated without the two interaction terms. The results of this are presented in Table 3.

This provides reasonable evidence that the "site of hospital-acquired infection" $(P=.06)$ and the "clinical service" $(P<.01)$ have a real effect on the prevalence and that "time period" $(P=.93)$ does not. It is therefore assumed that the underlying pattern of hospital-acquired infection over time is stable.

The number of hospital-acquired infections and the point prevalence, disaggregated by clinical service and site of infection, are presented in Table 4.

Five hundred fifty-three $(9.5 \%)$ patients were identified with a single site or with multiple sites of hospitalacquired infection from a population of 5,819 observed on the days of the surveys. The prevalence was found to be greatest among patients on adult and neonatal intensive care wards, followed by patients on surgical wards. The lowest prevalence was found among maternity patients. The most prevalent sites of hospital-acquired infection were the chest and multiple sites (MULTI) among adult patients in intensive care wards, the bloodstream (BS) and other sites (OTHER) among patients in neonatal intensive care wards, and surgical wounds (SW) among surgical patients. No urinary tract (UT) infections or surgical wound (SW) infections were found among patients in neonatal intensive care wards.

The results of the application of the formula to convert the prevalence to a measure of the cumulative incidence are presented in Table 5 . Estimates of the cumulative incidence and $95 \%$ confidence intervals are presented.

The estimates of the cumulative incidence based on $P$, $L A, L N$, and $I N T$ suggest that the highest rates of hospitalacquired infection will occur among adults in intensive care wards, then among patients in neonatal intensive care wards, and finally among surgical patients. Rates are lowest for maternity patients. UT would be the most frequently occurring single site of hospital-acquired infection, followed by SW, 
TABLE 4

Number of Cases and Point Prevalence (\%) for All Survey Periods

\begin{tabular}{|c|c|c|c|c|c|c|c|c|c|c|c|c|}
\hline \multirow{2}{*}{$\begin{array}{l}\text { Shte of } \\
\text { Hospltal- } \\
\text { Acquired } \\
\text { infection }\end{array}$} & \multicolumn{2}{|c|}{ Adult IC } & \multicolumn{2}{|c|}{ Medical } & \multicolumn{2}{|c|}{ Neonatal IC } & \multicolumn{2}{|c|}{ Maternity } & \multicolumn{2}{|c|}{ Surgleal } & \multicolumn{2}{|c|}{ All } \\
\hline & No. & (\%) & No. & (\%) & No. & $(\%)$ & No. & $(\%)$ & No. & $(\%)$ & No. & (\%) \\
\hline UT & 1 & $(0.66)$ & 44 & $(1.69)$ & & & 1 & $(0.12)$ & 42 & $(2.20)$ & 88 & (1.51) \\
\hline SW & 3 & (1.97) & 23 & $(0.88)$ & & & 5 & $(0.62)$ & 66 & (3.45) & 97 & (1.67) \\
\hline Chest & 11 & (7.24) & 47 & $(1.81)$ & 5 & (1.46) & 1 & $(0.12)$ & 33 & (1.73) & 97 & (1.67) \\
\hline Skin & 3 & $(1.97)$ & 27 & (1.04) & 5 & (1.46) & 2 & $(0.25)$ & 18 & $(0.94)$ & 55 & $(0.95)$ \\
\hline BS & 4 & (2.63) & 38 & (1.46) & 14 & $(4.09)$ & 2 & $(0.25)$ & 11 & $(0.58)$ & 69 & (1.19) \\
\hline OTHER & 2 & $(1.32)$ & 33 & (1.27) & 13 & $(3.80)$ & 8 & $(0.99)$ & 23 & $(1.20)$ & 79 & (1.36) \\
\hline MULTI & 7 & $(4.61)$ & 37 & $(1.42)$ & 3 & $(0.88)$ & 5 & $(0.62)$ & 16 & $(0.84)$ & 68 & (1.17) \\
\hline All & 31 & (20.39) & 249 & $(9.57)$ & 40 & $(11.70)$ & 24 & (2.96) & 209 & (10.93) & 553 & $(9.50)$ \\
\hline
\end{tabular}

TABLE 5

Predicted Cumulative Incidence (With 95\% Confidence Intervals) Based on Mean Values of $L A, L N$, ANd $I N T$

\begin{tabular}{|c|c|c|c|c|c|c|}
\hline \multirow{2}{*}{$\begin{array}{l}\text { Site of } \\
\text { Hospital- } \\
\text { Acquired } \\
\text { Infection }\end{array}$} & \multirow{2}{*}{ Adult IC } & Medlcal & Neonatal IC & Maternity & Surglcal & All \\
\hline & & $\% \quad\left(\mathrm{Cl}_{98}\right)$ & $\begin{array}{ll}\% & \left(\mathrm{Cl}_{98}\right)\end{array}$ & $\begin{array}{ll}\% & \left(\mathrm{Cl}_{98}\right)\end{array}$ & $\% \quad\left(\mathrm{Cl}_{98}\right)$ & $\begin{array}{ll}\% & \left(\mathrm{Cl}_{95}\right)\end{array}$ \\
\hline \multirow[t]{2}{*}{ UT } & 1.15 & 1.40 & & 0.03 & 2.54 & 1.46 \\
\hline & $(-0.85$ to 3.15$)$ & (1.14 to 1.67$)$ & & $(-1.97$ to 2.03$)$ & (2.27 to 2.81$)$ & (1.27 to 1.66$)$ \\
\hline \multirow[t]{2}{*}{ SW } & 0.96 & 0.51 & & 0.68 & 2.31 & 1.14 \\
\hline & $(-0.03$ to 1.95$)$ & $(0.12$ to 0.91$)$ & & (0.13 to 1.23$)$ & (1.96 to 2.66$)$ & $(0.89$ to 1.40$)$ \\
\hline \multirow[t]{2}{*}{ Chest } & 5.98 & 1.04 & 1.01 & 0.03 & 1.00 & 0.90 \\
\hline & (5.48 to 6.48$)$ & (0.56 to 1.51$)$ & $(0.12$ to 1.90$)$ & (-1.98 to 2.03$)$ & (0.62 to 1.37$)$ & (0.62 to 1.18$)$ \\
\hline \multirow[t]{2}{*}{ Skin } & 0.99 & 1.09 & 1.91 & 0.06 & 1.28 & 0.84 \\
\hline & $(-0.65$ to 2.63$)$ & ( 0.80 to 1.38 ) & (1.20 to 2.62$)$ & $(-2.02$ to 2.15$)$ & (0.94 to 1.63$)$ & (0.55 to 1.13$)$ \\
\hline \multirow[t]{2}{*}{ BS } & 1.06 & 1.77 & 4.84 & 0.24 & 0.48 & 0.88 \\
\hline & $(0.12$ to 2.01$)$ & (1.54 to 2.01 ) & $(4.29$ to 5.39$)$ & $(-0.76$ to 1.25$)$ & (0.11 to 0.85$)$ & (0.62 to 1.15$)$ \\
\hline \multirow[t]{2}{*}{ OTHER } & 0.77 & 1.00 & 2.33 & 1.24 & 0.88 & 0.79 \\
\hline & $(-0.78$ to 2.33$)$ & $(0.70$ to 1.30$)$ & (1.38 to 3.28$)$ & (0.95 to 1.53$)$ & (0.58 to 1.19$)$ & $(0.37$ to 1.21$)$ \\
\hline \multirow[t]{2}{*}{ MULTI } & 2.21 & 0.99 & 0.27 & 0.26 & 0.61 & 0.61 \\
\hline & (1.74 to 2.67$)$ & (0.55 to 1.43$)$ & $(-1.39$ to 1.93$)$ & $(-0.88$ to 1.40$)$ & (0.19 to 1.03$)$ & $(0.22$ to 1.00$)$ \\
\hline \multirow[t]{2}{*}{ All } & 11.33 & 7.29 & 8.78 & 1.76 & 8.41 & 6.33 \\
\hline & (11.00 to 11.65$)$ & (7.12 to 7.46$)$ & (8.30 to 9.25$)$ & (1.27 to 2.25$)$ & $(8.24$ to 8.58$)$ & (6.20 to 6.46$)$ \\
\hline
\end{tabular}

chest, OTHER, BS, and skin (SKIN). The lowest observed rate is for patients with MULTI. These results suggest that the overall cumulative incidence is $6.33 \%$ (95\% confidence interval, $6.20 \%$ to $6.46 \%$ ).

\section{DISCUSSION}

The prevalences reported here are consistent with those published in the international literature. A study in the United Kingdom ${ }^{11}$ found an overall prevalence of $9 \%$. A survey of 123 hospitals conducted in Spain ${ }^{12}$ found a prevalence of $9.9 \%$, a survey of 15 hospitals in Norway ${ }^{13}$ found a prevalence of $9.0 \%$, and a survey of 47 hospitals in 14 countries ${ }^{14}$ found a prevalence of $8.7 \%$. There are limited published data on how prevalence varies by service and site of hospital-acquired infection, but patients in intensive care beds have consistently demonstrated the highest prevalence and the prevalence is generally higher among surgical than among medical patients. ${ }^{1}$ 
The true values for INT were not known and so several assumptions were made to allow for the estimation of INT. In particular, it was assumed that on the day of the survey, patients with a hospital-acquired infection were halfway through a course of treatment. This relies on a nested assumption that the variable that describes the number of days a patient has been in receipt of treatment, for a defined treatment program, was normally distributed. If this were the case, then the midpoint (the average) would be the most appropriate value to use. The underlying nature of the probability distribution for this variable was not known, but there was nothing to suggest it would not be normally distributed.

The limitations of the formula used to estimate cumulative incidence from prevalence data have been discussed by those who developed it ${ }^{7}$ and those who have applied it. ${ }^{8,9}$ The formula relies on infections occurring independently of each other and the probability of acquiring subsequent infections must not be influenced by existing infections. Whether this is the case for these patient groups has not been explored. It is also argued that the prevalence is generally greater than any estimates of the cumulative incidence and so the product of

$$
\left(\frac{L A}{L N-I N T}\right)
$$

should be less than 1 . This is not true for several of the results derived from this patient group, specifically adult patients in the intensive care ward with UT infections, patients in the neonatal intensive care ward and medical patients with skin and BS infections, maternity patients with SW and other infections, and surgical patients with UT and skin infections. The reason for some of these findings might be the small numbers of hospital-acquired infections (eg, three of these estimates were derived from data on five or fewer cases of hospital-acquired infection). However, for some of the other findings, it might be the case that the values for $L N-I N T$ and $L A$ are accurate. For example, all patients in intensive care wards will be severely ill and other, more serious comorbidities may extend the length of stay for all patients sufficiently to negate any differences in length of stay due to hospital-acquired infection. Also, maternity and surgical patients are likely to be younger and more fit than other patient groups (eg, medical patients) and therefore less likely to have their stay prolonged by hospital-acquired infection compared with those without hospital-acquired infection. They may be better able to deal with a hospital-acquired infection in their home with the support of family and community care services. These arguments do not apply to medical patients with BS infections and infections of the skin. It would be expected that $L N$ - INT would exceed $L A$ for this patient group. The answer may lie in the values used in the formula. Although data on $L A$ and $L N$ were collected from reliable sources using a recognized method, the values for $I N T$ were based on several assumptions. It may be that these assumptions were inappropriate for this patient group.
Care should be taken when comparing the estimates of incidence derived in this study with rates reported in other incidence studies because of differences in time period, country, population surveyed, and survey methods. With this in mind, the estimates of the cumulative incidence compare favorably with rates reported in the literature. The Study on the Efficacy of Nosocomial Infection Control (SENIC) conducted in the United States found an overall incidence rate of $5.7 \%{ }^{15} \mathrm{~A}$ study of the socioeconomic burden of hospital-acquired infection conducted in the United Kingdom ${ }^{16}$ found the overall incidence of hospital-acquired infection to be $7.8 \%$. From this study, rates were as follows: UT, $2.7 \%$; chest, $1.2 \%$; SW, $1.0 \%$; BS, $0.1 \%$; skin, $0.6 \%$; OTHER, $0.8 \%$; and MULTI, $1.4 \%$. With the exception of UT infection, BS infection, and MULTI, the confidence intervals for the rates reported here encompass the rates reported from the United Kingdom study. For BS infection, case mix variation may explain the differences between the findings. The United Kingdom study excluded neonates, but in this study they were found to have the highest rate for BS infection. For UT infection, the rates reported here are lower than those of the United Kingdom study, but this was found to be the most frequently occurring site of hospital-acquired infection in both studies.

One of the strengths of this work is the degree to which the data are disaggregated. Insights are provided into rates of hospital-acquired infection for many patient groups with the most common sites of hospital-acquired infection. To conduct incidence studies for such a wide range of patients would be an expensive and time-consuming exercise. This work has used all of the data available from the seven routine prevalence surveys and appears to be a cost-effective method for predicting the likely cumulative incidence of hospital-acquired infection in Auckland District Health Board Hospitals in New Zealand. The estimates of the cumulative incidence reported here can be used for further research and modeling that will contribute to the formulation of appropriate local policies for the control of hospital-acquired infection in New Zealand.

\section{REFERENCES}

1. Plowman RP, Graves N, Roberts JA. Hospital Acquired Infection. London: Office of Health Economics; 1997.

2. Haley RW. Managing Hospital Infection Control for Cost-effectiveness: A Strategy for Reducing Infectious Complications. Chicago: American Heart Association; 1986:

3. Plowman R, Graves N, Esquivel J, Roberts J. An economic model to assess the cost and benefits of the routine use of silver alloy coated urinary catheters to reduce the risk of urinary tract infections in catheterised patients. J Hosp Infect 2001;48:1:33-42.

4. Wenzel RP. Nosocomial candidemia: risk factors and attributable mortality. Clin Infect Dis 1995;20:6:1531-1534.

5. Haley R. Cost benefit analysis of infection control activities. In: Brachman P, Bennett J, eds. Hospital Infections. Boston: Little, Brown; 1992:507-532.

6. Nicholls T, Morris A. Nosocomial infection in Auckland Healthcare hospitals. $N Z$ Med J 1997;110:1050:314-316.

7. Rhame F, Sudderth W. Incidence and prevalence as used in the analysis of the occurrence of nosocomial infections. Am J Epidemiol 1981;113:111 .

8. Delgado-Rodriguez M, Cueto-Espinar A, Rodriguez-Contreras-Pelayo R, Galvez-Vargas R. A practical application of Rhame and Sudderth's formula on nosocomial infection surveillance. Rev Epidemiol Sante Publique 1987:35:482-487

9. Gastmeier P, Brauer H, Sohr D, et al. Converting incidence and preva- 
lence data of nosocomial infections: results from eight hospitals. Infect Control Hosp Epidemiol 2001;22:1:31-34.

10. Garner J, Jarvis R, Emori T, Horan T, Hughes J. CDC definitions for nosocomial infections, 1988. Am J Med 1988;16:28-40.

11. Emmerson A, Entstone J, Griffin M, Kelsey M, Smyth E. The second national prevalence survey of infection in hospitals: overview of the results. J Hosp Infect 1996;32:3:175-190.

12. EPINE Working Group. Prevalence of hospital infections in Spain. $J$ Hosp Infect 1992;20:1-13.

13. Hovig B, Lystad A, Opsjon H. A prevalence survey of infections amongst hospitalised patients in Norway. NIPH Annals 1981;4:49-60.

14. Mayon-White RT, Ducel G, Kereselidze T, Tikomirov E. An internation- al survey of the prevalence of hospital acquired infection. J Hosp Infect $1988 ; 11$ (suppl A):43-48.

15. Haley R, White J, Culver D, et al. The efficacy of infection surveillance and control programmes in preventing nosocomial infections in US hospitals. Am J Epidemiol 1985:121:182-205.

16. Plowman RP, Graves N, Griffin MAS, et al. The rate and cost of hospitalacquired infections occurring in patients admitted to selected specialties of a district general hospital in England and the national burden imposed. J Hosp Infect 2001;47:198-209.

17. Lang S. Guide to Pathogens and Antibiotic Treatment. Auckland, New Zealand: Adis International; 2001.

\section{APPENDIX}

The $95 \%$ confidence intervals were calculated for the estimates of the cumulative incidence using the formula

$$
S E(\log I)=\sqrt{\frac{1}{P^{2}}}\left(\frac{P(\operatorname{var} P)}{n}\right)+\frac{1}{L A^{2}}\left(\frac{\sigma L A^{2}}{n 1}\right)+\frac{1}{D^{2}}\left(\frac{\sigma D^{2}}{n 2}\right),
$$

where $I=$ incidence; $P=$ prevalence; $\operatorname{var} P=$ the variance of $P ; n=$ the number of observations on which $P$ was based; $L A=$ average length of stay of all patients; $n 1=$ the number of observations on which $L A$ was based; $L N=$ average length of stay of patients who acquire one or more nosocomial infections; $I N T$ = average interval between admission and onset of the first nosocomial infection for those patients who acquire one or more nosocomial infections; $D=L N-I N T$; and $n 2=$ the number of observations on which $D$ was based.

The anti-log of $S E(\log I)$ was taken and multiplied by 2 to derive $95 \%$ confidence intervals for the estimate of $I$. 